\title{
Soluble P-selectin for the diagnosis of lower extremity deep venous thrombosis
}

\author{
Frank C. Vandy, MDa, Cathy Stabler, RN ${ }^{\mathrm{a}}$, Anna M. Eliassen, BS ${ }^{\mathrm{a}}$, Angela E. Hawley, BS ${ }^{\mathrm{a}}$, \\ Kenneth E. Guire, MS ${ }^{b}$, Daniel D. Myers, DVM ${ }^{a}$, Peter K. Henke, MD ${ }^{a}$, and Thomas W. \\ Wakefield, MDa \\ aSection of Vascular Surgery, Department of Surgery, University of Michigan, Ann Arbor, Mich \\ ${ }^{b}$ Department of Biostatistics, School of Public Health, University of Michigan, Ann Arbor, Mich
}

\section{Abstract}

Objective-Although duplex ultrasound is the standard for the diagnosis of lower extremity deep venous thrombosis (LE-DVT), imaging is not always available. The use of D-dimer can exclude (high-sensitivity), but not rule in (low-specificity) LE-DVT. Previously, we demonstrated that soluble P-selectin (sP-sel) in combination with the Wells score, establishes the diagnosis of LEDVT with a specificity of $96 \%$ and a positive predictive value of $100 \%$. In order to validate our previous results, we applied the model to a separate but similar patient cohort. Additionally, we analyzed the role of biomarkers for diagnosing upper extremity DVT (UE-DVT).

Methods-Between April 2009 and March 2012, all patients presenting for a duplex ultrasound exam with concern of DVT were screened. Demographics, clinical data, D-dimer, sP-sel, Creactive protein, a disintegrin and metalloproteinase with a thrombospondin type 1 motif, member 13, and von Willebrand factor levels were prospectively collected in 279 patients (234 LE-DVT, 45 UE-DVT). Continuous and categorical variables among patients with DVT were compared with patients without DVT. The diagnostic sensitivity, specificity, positive predictive value, and negative predictive value were then calculated using our previously derived cut points to rule in or exclude DVT.

Results-Among 234 patients evaluated for LE-DVT, 112 (48\%) patients had a confirmed LEDVT with significant differences in all biomarkers. When Wells score 22 , sP-sel could rule in LEDVT with a specificity of $97.5 \%$ and a positive predictive value of $91 \%$, which was more accurate than Wells score $\geq 2$ and D-dimer (specificity, 65\%; positive predictive value, 69\%). When Wells score was $<2$, D-dimer was superior to $\mathrm{sP}$-sel for excluding the diagnosis of LE-DVT (sensitivity,

Copyright $(2013$ by the Society for Vascular Surgery. Published by Elsevier Inc. All rights reserved.

Reprint requests: Thomas W. Wakefield, MD, James C. Stanley Professor in Vascular Surgery, Section Head - Vascular Surgery, CVC - 5463, 1500 E. Medical Center Rd, Ann Arbor, MI 48109-5867 (thomasww@umich.edu). .

AUTHOR CONTRIBUTIONS Conception and design: FV, CS, KG, DM, PH, TW

Analysis and interpretation: FV, AE, KG, TW

Data collection: CS, AH

Writing the article: FV, AE, TW

Critical revision of the article: FV, CS, AH, KG, DM, PH, TW

Final approval of the article: FV, PH, TW

Statistical analysis: FV, KG

Obtained funding: TW

Overall responsibility: FV, TW

Author conflict of interest: none.

Presented at the Twenty-fourth Annual Meeting of the American Venous Forum, Orlando, Fla, February 8-11, 2012.

The editors and reviewers of this article have no relevant financial relationships to disclose per the Journal policy that requires reviewers to decline review of any manuscript for which they may have a conflict of interest. 
98\%; negative predictive value, $95 \%$ vs sensitivity, $91 \%$; negative predictive value, $79 \%$ ). The use of additional biomarkers did not increase accuracy. Had imaging not been available, we could have correctly ruled in or ruled out LE-DVT in 29\% (67/234) of patients. The use of sP-sel in UEDVT was nondiagnostic.

Conclusions-We demonstrate that when Wells score $\geq 2, \mathrm{sP}$-sel is an excellent biomarker to rule in LE-DVT. Different from our previous study, D-dimer and a Wells score <2 was most sensitive at excluding a diagnosis of LE-DVT. Combined, Wells score, sP-sel, and D-dimer can both rule in and exclude LE-DVT in approximately one-third of patients.

Venous thromboembolism (VTE) is a substantial health problem in the United States, with an estimated incidence of up to 3 per 1000 people annually. ${ }^{1}$ The morbidity and mortality of undiagnosed VTE remains high, as nearly $60 \%$ of undiagnosed deep venous thrombosis (DVT) eventually leads to pulmonary embolism (PE), causing over 50,000 deaths per year. ${ }^{2,3}$ Therefore, it is important to establish the diagnosis and initiate anticoagulation therapy, when appropriate, in a timely manner. However, establishing a diagnosis of DVT based on clinical presentation alone is unreliable, as none of the signs or symptoms in isolation are diagnostic. This predicament has led to the development of clinical scoring systems such as the Wells criteria (Table I) that incorporate signs, symptoms, and risk factors to categorize patients as low, moderate, or high probability for DVT and thus help determine the need for further workup. ${ }^{4}$

Currently, the only biomarker used in the diagnosis of DVT is plasma D-dimer. D-dimer, a product of fibrin degradation, is a sensitive biomarker that, when low or absent, can be used to exclude, or "rule out" the diagnosis of DVT in patients with a low Wells score. ${ }^{4}$ However, this biomarker is not specific for DVT, as it is frequently elevated in the setting of nonspecific inflammation such as cancer, pregnancy, surgery, and trauma. ${ }^{5}$ Because of the low specificity of D-dimer, elevated levels alone cannot be used to rule in the diagnosis of DVT, and in such instances, an imaging study is needed for definitive diagnosis. The current gold standard for diagnosis of DVT is compression duplex ultrasound. ${ }^{6}$ However, the availability of this diagnostic modality is limited at smaller medical centers, outpatient settings, and on nights and weekends. In these circumstances, a chemical or laboratory diagnosis to "rule in" the diagnosis of DVT without having to rely on duplex ultrasound would be helpful. Using the biology of platelet and leukocyte activation, inflammation, and thrombosis, our laboratory has identified soluble P-selectin (sP-sel), soluble E selectin (sEsel), and microparticles (MP) as potential novel biomarkers to make the diagnosis of DVT. ${ }^{7}$ Of these, $\mathrm{sP}$-sel has shown the greatest promise.

P-selectin is a cell adhesion molecule that is stored in the alpha granules of platelets and the Weibel-Palade bodies of endothelial cells. ${ }^{8}$ Upon platelet and endothelial cell activation, Pselectin molecules are translocated to the cell surface and released into the circulation in its soluble form. Through a series of downstream signaling events, sP-sel mediates thrombosis, hemostasis, and the platelet-endothelial interaction via the release of procoagulant microparticles, platelet thromboxane A2, and platelet metalloproteinase-2. ${ }^{9-16}$ Given these procoagulant properties of sP-sel, recent studies have focused on the clinical significance of its role in VTE, demonstrating elevated plasma levels of sP-sel in patients with acute VTE. ${ }^{17-21}$

In a 2005 pilot study, we reported that the combination of MP, sP-sel, and D-dimer resulted in $73 \%$ sensitivity and $81 \%$ specificity for diagnosing VTE. ${ }^{19}$ In follow-up, we established that a combination of Wells score $\geq 2$ and a sP-sel $>90 \mathrm{ng} / \mathrm{mL}$ could rule in the diagnosis of lower extremity DVT (LE-DVT) with a specificity of $95 \%$ and a positive predictive value of $100 \%$. Conversely, a Wells score $<2$ and a $\mathrm{sP}-\mathrm{sel}<60 \mathrm{ng} / \mathrm{mL}$ could rule out the diagnosis with a sensitivity of $99 \%$ and a negative predictive value of $96 \% .{ }^{22}$ In comparison, D-dimer 
only had a sensitivity of $93 \%$ and negative predictive value of $81 \%$ for ruling out DVT. However, in our previous study, our investigational cohort of patients served as our derivation sample to establish our diagnostic cut points for sP-sel. Therefore, we set forth to validate our previously derived $\mathrm{sP}$-sel diagnostic rule with a separate cohort of patients. We hypothesized that a Wells score $\geq 2$ and a sP-sel $>90 \mathrm{ng} / \mathrm{mL}$ could rule in the diagnosis of LE-DVT, while a Wells score $<2$ and a sP-sel $<60 \mathrm{ng} / \mathrm{mL}$ could rule out the diagnosis. In utilizing this diagnostic rule, a sP-sel level between 60 and $90 \mathrm{ng} / \mathrm{mL}$ was indeterminate and considered non-diagnostic to either rule in or rule out thrombosis.

\section{METHODS}

The University of Michigan Institutional Review Board approved this protocol. This was a prospective study. From April 2009 through March 2012, all inpatients and outpatients sent to our diagnostic vascular ultrasound lab with signs or symptoms concerning for acute LEor upper extremity DVT (UE-DVT) were screened for enrollment. Following informed consent, patients underwent a UE or LE compression ultrasound DVT scan. A registered nurse study coordinator blinded to the results of the duplex then performed a clinical assessment to determine a Wells score ${ }^{4}$ and a Caprini DVT risk score. ${ }^{23}$ Demographic variables were also recorded. Blood was drawn at this time to be analyzed in our laboratory for the following biomarkers: sP-sel, D-dimer, C-reactive protein(CRP), vonWillebrandfactor(vWf), anda disintegrin and metalloproteinase with a thrombospondin type 1 motif, member 13 (ADAMTS-13).

\section{Patient selection}

Patients were considered ineligible for enrollment if they were less than 18 years of age, were unable to give informed consent, or had an isolated calf vein DVT. Isolated calf DVTs were excluded in order to be consistent with our previous publication, evaluate only the most significant DVTs, and eliminate technical error associated with identifying isolated calf vein DVT. Regarding LE-DVT, only patients with DVTs located in the popliteal vein and more proximally were recruited. Patients who were diagnosed with both above- and below-knee DVTs were included as long as there was at least a popliteal or more proximal component-thus, isolated calf-level thrombi were excluded. Patients with isolated UE-DVT were included. Study inclusion, and hence analysis, was limited to only those patients presenting with signs or symptoms of acute DVT. Additional exclusionary criteria included patients with superficial thrombosis, indeterminate scans, chronic thrombus by ultrasound, pregnant or nursing patients, line-associated fibrin sheath in the absence of clot, and patients on therapeutic anticoagulation. Furthermore, in order to determine if there were differences in biomarkers between LE-DVT and UE-DVT, those patients presenting with simultaneous UE- and LE-DVT were excluded. Patients on prophylactic anticoagulation with either subcutaneous heparin or low-molecular-weight heparin (LMWH), as well as patients on immunosuppression medications were included in this study.

\section{Compression ultrasound}

In those patients with DVT, the extent of DVT was grossly quantitated. The leg was divided into eight segments corresponding to LE venous anatomy (Fig 1). We do not routinely image the common iliac or inferior vena cava. Segments were then totaled, providing a DVT extent score ranging from 1 to 8 . In addition, consideration was given to the location of the LE-DVT. DVTs involving the iliac and/or common femoral vein were referred to as proximal LE-DVT, while DVTs involving the femoral and/or popliteal vein in association with or without DVT in the peroneal, anterior tibial, or posterior tibial veins and great saphenous vein were designated distal LE-DVT. 


\section{Blood analysis}

At the time of enrollment/consent, all blood samples were collected in sodium citrate tubes, centrifuged for 10 minutes at $2000 \mathrm{~g}$ at $4^{\circ} \mathrm{C}$ (except for plasma samples to determine ADAMTS13 that underwent extra centrifugation [2 minutes at $15,000 \mathrm{~g}$ at $25^{\circ} \mathrm{C}$ ]), plasma was drawn off, snap-frozen in liquid nitrogen, and stored at $-70^{\circ} \mathrm{C}$. Commercially available enzyme-linked immunosorbent assays were used to evaluate circulating sP-sel levels (Hyphen Biomed, Neuvillesur-Olse, France), D-dimer (Hyphen Biomed), and CRP levels (US Biological, Swampscott, Mass). They were reported in ng/mL (sP-sel), mg/L (D-dimer), and $\mu \mathrm{g} / \mathrm{mL}$ (CRP). Plasma VWF activity (Corgenix Inc, Broomfield, Colo) was reported as a relative percentage $(\%)$ as compared with pooled normal plasma. Samples were prepared according to the manufacturers' instructions, run in duplicate, and read on an Elx808 plate reader (Biotek, Winooski, Vt) at $450 \mathrm{~nm}$ wavelength. These analyses were performed in a blinded fashion, as the samples were identified by a numbering system. ADAMTS-13 activity was determined by the slightly modified FRETS-VWF73 assay. ${ }^{24,25}$

As previously described, sP-sel levels greater or equal to $90 \mathrm{ng} / \mathrm{mL}$ were used to rule in the diagnosis of DVT while an sP-sel less than $60 \mathrm{ng} / \mathrm{mL}$ was used to rule out the diagnosis of DVT. Based on manufacturer recommendations as well as our previous work, a D-dimer value greater or equal to $0.5 \mathrm{mg} / \mathrm{L}$ was considered abnormally high. vWf and ADAMTS-13 were measured in an activity percentage (\%) as compared with pooled normal plasma.

\section{Statistical analysis}

Statistical analysis was performed comparing those patients with DVT and those without (SAS statistical software, Cary, NC). Continuous variables were compared using a Student's $t$-test, while dichotomous categorical variables were compared using a $\chi^{2}$ analysis. The relationship between biomarkers and demographics (body mass index, age) as well as biomarkers and extent of DVT were assessed using Spearman correlation coefficients. A secondary analysis was conducted comparing biomarker levels with respect to location of DVT, UE versus LE. Sensitivity, specificity, positive predictive value, and negative predictive value were determined using our previously derived sP-sel and D-dimer diagnostic values.

As previously described, logistic regression models were once again used to study the various independent biomarker and clinical variables (alone and in combination) in their ability to diagnose DVT. Receiver-operating characteristic (ROC) curves were constructed using sensitivity versus 1-specificity to assess the diagnostic value of two-, three-, or fourvariable models of biomarkers combined or a biomarker combined with the clinical factors. We defined the optimal biomarker, either alone or in combination, as that presenting the best combination of receiver-operating characteristic, accuracy, sensitivity, and specificity.

\section{RESULTS}

Between April 9, 2009 and March 30, 2012, 2791 patients were screened for enrollment into this study. Two thousand, four hundred eighty patients did not meet inclusion criteria or declined participation, leaving 311 patients considered eligible for enrollment. Following enrollment, 12 patients were unable to undergo successful phlebotomy, three electively withdrew, and 17 had blood samples that were unsuccessfully analyzed by our laboratory, leaving 279 patients for analysis, 234 patients in the LE-DVT cohort, and 45 patients in the UE-DVT cohort. (Fig 2)

Patients with an LE-DVT were significantly more likely to be older, be male, have had a previous DVT, have active cancer or a history of cancer, and be on prophylactic anticoagulation. Patients in the LE-DVT cohort were also more likely to be of inpatient 
status, as opposed to outpatient and had a significantly higher Caprini risk score as well as a higher Wells score (Table II, $A$ ). Conversely, those patients with UE-DVT were only more likely to be male and of inpatient status (Table II, $B$ ). In patients who did have an LE-DVT, the thrombus burden was considerable, with an average DVT extent score of 4.2.

Five biomarkers were compared between those patients who had an ultrasound-confirmed DVT and those patients who had a suspected DVT. In LE-DVT, there was a significant difference between groups for each biomarker analyzed, favoring higher levels in those patients with a DVT (Table III, $A$ ). As expected, levels of ADAMTS-13 demonstrated a negative correlation with increasing levels of vWf activity, with ADAMTS-13 activity significantly lower in those patients with DVT.

In patients with UE-DVT, only Caprini and Wells score, D-dimer, and CRP were found to be significantly higher. There was no significant difference in $\mathrm{sP}$-sel between suspected and confirmed UE-DVT (Table III, $B$ ). However, when biomarkers were compared by location of DVT, there were significantly higher levels of sP-sel in patients with confirmed LE-DVT than confirmed UE-DVT. There was no significant variation between the remaining biomarkers with respect to DVT location (Table IV).

In patients with LE-DVT, Spearman's rank order correlations were used to test the association between levels of biomarkers and demographic characteristics as well as the DVT extent score. There was no statistical difference between biomarkers and gender or body mass index; however, with increasing age, $\mathrm{vWf}$ was found to increase (coefficient $0.31 ; P=.001$ ) with a reciprocal decrease in ADAMTS-13 (coefficient $-0.3 ; P=.007$ ). DVT extent score was seen to positively correlate with D-dimer (coefficient $0.27 ; P=.003$ ), sPsel (coefficient $0.21 ; P=.026$ ), and CRP (coefficient $0.26 ; P=.006$ ), and Wells score (coefficient $0.18 ; P=.04$ ). When we compared the level of biomarkers among patients who had proximal LE-DVT and distal LE-DVT, there was no statistical difference. Furthermore, using pairwise comparisons, in those patients with both proximal and distal LE-DVT, the biomarkers measured trended but were not significantly higher than in LE-DVTs involving just the proximal or the distal segments alone (Table V).

A subset analysis comparing those patients who developed an LE-DVT while on prophylactic anticoagulation $(\mathrm{n}=17)$ with those patients not on prophylactic coagulation $(\mathrm{n}$ $=95$ ) revealed no difference in demographics other than inpatient status. Furthermore, there was no significant difference in Wells score or biomarkers (Table VI).

When our previously derived diagnostic parameters for LE-DVT were applied to the current population of patients, a sP-sel level of $\geq 0 \mathrm{ng} / \mathrm{mL}$ and a Wells score of $\geq 2$ was able to rule in the diagnosis of LE-DVT with a specificity of $97.5 \%$, yielding a positive predictive value of $91 \%$. Conversely, when a D-dimer level of $\searrow 0.5 \mathrm{mg} / \mathrm{L}$ and a Wells score of $\geq 2$ was used to make a diagnosis of LE-DVT, the specificity was $65.5 \%$ for ruling in LE-DVT, and the positive predictive value was only $69 \%$. However, a D-dimer $₫ 5.5 \mathrm{mg} / \mathrm{L}$ and a Wells score $<2$ effectively ruled out LE-DVT with a sensitivity of $98 \%$ and a negative predictive value of $95 \%$. sP-sel $\$ 60 \mathrm{ng} / \mathrm{mL}$ and Wells score $<2$ was inferior to D-dimer for ruling out LEDVT, yielding a sensitivity of $91 \%$ and a negative predictive value of only $79 \%$ (Table VII). Using the previously derived diagnostic rules, 29\% (67/234) of patients were either ruled in or ruled out for LE-DVT.

As previously described, a logistic regression model was used to determine the best combination of biomarkers or biomarkers and clinical variables to make a diagnosis of LEDVT. Repeat analysis on the current cohort revealed that a four-variable model including sP-sel, D-dimer, and Wells score, as well as a Caprini risk score gave a diagnostic accuracy of $84.2 \%$ with a sensitivity $80.5 \%$ and a specificity of $87.6 \%$ (ROC, . $918 ; P<.001$ ). This 
model was the best combination for ruling in a diagnosis of LE-DVT, and the inclusion of additional biomarkers into this model did not increase diagnostic yield.

\section{DISCUSSION}

This study represents a validation of our previously derived sP-sel diagnostic parameters for making a laboratory diagnosis of LE-DVT. Indeed, we once again demonstrated that a Wells score of $\geq 2$ and sP-sel $\geq 90 \mathrm{ng} / \mathrm{mL}$ was accurate at ruling in the diagnosis of LE-DVT with a positive predictive value of $91 \%$ and a specificity of 97.5 . Thus, sP-sel continues to be superior to D-dimer in making the diagnosis of LE-DVT. Conversely, our previous efforts demonstrated a Wells score $<2$ and a sP-sel $<60 \mathrm{ng} / \mathrm{mL}$ to be superior to D-dimer for ruling out LE-DVT. However, in the current study, this was not the case, as Wells score $<2$ and Ddimer levels less than $0.5 \mathrm{mg} / \mathrm{L}$ excluded the diagnosis of LE-DVT with a negative predictive value of $95 \%$ and sensitivity of $98 \%$. Using a combination of Wells score, sP-sel, and D-dimer, a conclusive diagnosis ruling in or ruling out LE-DVT could have been made in 29\% (67/234) of cases without the use of additional imaging. This is consistent with our previous study in which $32 \%$ of cases could have been diagnosed with or without LE-DVT using just the clinical exam and laboratory values. ${ }^{22}$

The current validation cohort differed slightly from the previous derivation cohort. In the current study, we allowed the inclusion of patients on immunosuppression and prophylactic anticoagulation. Continued inclusion of inpatients, patients with active cancer, and those with previous VTE gives the current study and use of sP-sel a larger "real-world" application. The use of some immunosuppressant medications has been shown to enhance systemic thrombogenicity as well as elevate levels of sP-sel. ${ }^{26,27}$ As $15 \%$ of our study population was on some form of immunosuppression, we found it prudent to include these patients. However, in univariate analysis, there was no difference in the number of patients on immunosuppression that had a duplex-confirmed LE-DVT compared with those who did not have an LE-DVT. Furthermore, in patients without LE-DVT, there was no statistical difference in sP-sel among those patients taking or not taking immunosuppressive medications $(56.3 \mathrm{ng} / \mathrm{mL}$ vs $54.3 \mathrm{ng} / \mathrm{mL} ; P=.77)$.

Also unique to the study was the inclusion of patients on prophylactic anticoagulation. The effects of prophylactic anticoagulation (subcutaneous heparin three times daily; LMWH once daily) on sP-sel have not been well studied. Conversely, the effect of therapeutic anticoagulation, both oral vitamin $\mathrm{K}$ antagonists and intravenous heparin, has been reported. As demonstrated by Gremmel et al, both levels of sP-sel and D-dimer were significantly influenced by the use of oral anticoagulants, decreasing with initiation to normal levels and rising upon cessation. ${ }^{28}$ Likewise, upon therapeutic infusions of enoxaparin, Kvasnička et al demonstrated a significant drop in circulating levels of sP-sel within 20 minutes. ${ }^{29} \mathrm{In}$ a small group of patients outside of the current study, we measured selected biomarkers in the presence of therapeutic anticoagulation. Although small in number, we too demonstrated a sizeable drop in both sP-sel and D-dimer in those patients on therapeutic anticoagulation (LMWH - one, intravenous unfractionated heparin - four). However, in those patients on prophylactic anticoagulation, there was no significant difference in sP-sel or D-dimer. Thus, the efficacy of sP-sel or D-dimer as a diagnostic test to diagnose LE-DVT should not be altered in those patients on prophylactic anticoagulation.

In a secondary analysis, we evaluated the demographics and biomarker marker profiles of those patients with UE-DVT. In contrast to LE-DVT, there was no difference in levels of sPsel in those patients with and without UE-DVT. This sample size was much smaller in number than the LE-DVT cohort. Yet there was still a significant difference in D-dimer level among those patients with and without UE-DVT. However, of the 32 patients who 
developed a UE-DVT, 18 were associated with an indwelling central venous catheter (CVC). The presence of a CVC is the most powerful independent predictor of UE-DVT (odds ratio, 9.7; 95\% confidence interval, 7.8-12.2), and 55\% of UE-DVT occur in patients who have a CVC or have had one within 30 days of the thrombosis diagnosis. ${ }^{30}$ Expectedly, the pathophysiology of UE-DVT in the setting of an indwelling CVC is different than spontaneous DVT in the LE. The presence of a CVC may lead to the development of a circumferential fibrin sheath from the tip of the catheter to its insertion point. ${ }^{31}$ While fibrin sheaths may be asymptomatic, they predispose the vein to the development of thrombus on the CVC. In this fashion, we speculate the regulatory function of sP-sel may be limited to the platelet interface, as the sP-sel:PSGL-1 interaction at the endothelial cell surface may not play a dominant role in thrombus formation in the setting of a CVC. This may account for the variability in UE sP-sel values and lower levels as well as no significant difference between those patients with and without UE-DVT. Furthermore, the veins in the UE are shorter and have less endothelial surface on which to form clot. ${ }^{31}$ Thus, it is not surprising that lower levels of sP-sel were noted in UE-DVT. Interestingly, in those patients with a UEDVT, there was no difference between D-dimer, sP-sel, and CRP between patients that did and did not have a UE-CVC. However, we noted a significant decrease in ADAMTS-13 ( $72 \%$ activity vs $100 \%$ activity; $P=.01$ ) in those patients with a CVC. These findings may support a greater role for platelet-mediated thrombus growth independent of the endothelium in the setting of a foreign body such as a CVC. Based on these observations, we cannot advocate for the use of sP-sel in the diagnosis of UE-DVT. Given the small sample size of those patients with UE-DVT who did not have an indwelling CVC, it is difficult to draw any substantial statistical conclusions.

Our data suggest that thrombus burden, or amount of clot, positively correlates with sP-sel, D-dimer, CRP, and Wells score. Furthermore, those patients who had both proximal and distal LE-DVTs had higher levels of sP-sel, D-dimer, CRP, and Wells score than those patients who had only proximal or distal LE-DVTs. However, only the Wells score and not biomarker levels were shown to be significantly different in these levels. This would suggest that the sP-sel diagnostic efficacy is greatest in identifying extensive LE-DVTs. Other than thrombus extent, in the setting of DVT, it is unknown which factors, if any, lead to greater levels of sP-sel. As already mentioned, prophylactic anticoagulation, as well as body mass index, age, and gender does not appear to contribute to a difference in levels of sP-sel. While we did observe a difference in sP-sel between UE- and LE-DVT, this may have been the result of the pathophysiologic mechanism behind the DVT itself rather than the location of the DVT. We can speculate that the sP-sel levels may be affected by the chronicity of the clot, with sP-sel levels measured within hours of clot development being different than those measured at a later interval. Alternatively, the mass of thrombus in the UE is certainly lower than in the LE in most circumstances.

This study does have limitations. We were successful in once again making a diagnosis ruling in or ruling out LE-DVT in $29 \%$ of patients. However, our diagnostic rules only capture patients with very high levels of sP-sel. Like-wise, our predetermined diagnostic parameter for D-dimer only excludes patients with very low levels. There is a large portion of our study population ( $70 \%)$ that has a D-dimer level too high to be excluded from DVT but an SP-sel level too low to be ruled in for DVT. However, in order to increase the diagnostic yield for ruling in DVT, we would have to lower the diagnostic threshold or cutpoint of sP-sel, decreasing the specificity, and thus leading to a higher rate of false positives. This limitation does not diminish the importance of being able to rule in or rule out the diagnosis of LE-DVT in up to one-third of patients in whom ultrasound may not be available. 
An assay for sP-sel has yet to become available for clinical use, and current assays are not conducive for individual use. Our data only represent a midterm analysis. This study continues with active recruitment, and current data are subject to change. The use of sP-sel in the diagnosis of DVT is still in its infancy. Much remains unknown about factors than can cause elevations and reductions in sP-sel, both of which can limit the diagnostic accuracy of this assay. Finally, while some have suggested that the D-dimer values should be ageadjusted, our age ranges were relatively consistent for both cohorts, UE- and LE-DVT. ${ }^{32}$ Our decision to not age-adjust D-dimer levels reflected the intent to evaluate the test as it is currently used in most medical centers.

Despite these limitations, this study has clear advantages. This is the largest study to evaluate the role of sP-sel in the diagnosis of LE-DVT. We have validated a previously derived diagnostic rule in a "real-world" population. Furthermore, as in our previous study, a laboratory diagnosis ruling in or ruling out DVT was possible in almost $30 \%$ of cases without the need for additional imaging.

\section{CONCLUSIONS}

Compression ultrasound is the current standard of care for the diagnosis of DVT. Our results do not question or advocate a change in the current standard of care. However, ultrasound is not always available, especially on the weekend, at night, or in smaller hospitals. In circumstances when ultrasound in not available, sP-sel takes advantage of the biology associated with thrombosis and inflammation and adds to the widely used D-dimer assay and the Wells criteria for the diagnosis of DVT. These biomarkers in association with the clinical exam show early promise of making a laboratory diagnosis of LE-DVT.

\section{Acknowledgments}

The authors would like to acknowledge the efforts of Nichole Baker, RVT, for data entry as well as the laboratories of Johanna A. Kremer Hovinga, MD, and Denisa D. Wagner, PhD, for their contributions in blood sample analysis.

Supported by NIH grant HL095091 to T.W.W.

\section{REFERENCES}

1. Reitsma PH, Versteeg HH, Middeldorp S. Mechanistic view of risk factors for venous thromboembolism. Arterioscler Thromb Vasc Biol. 2012; 32:563-8. [PubMed: 22345594]

2. Appelman PT, De Jong TE, Lampmann LE. Deep venous thrombosis of the leg: US findings. Radiology. 1987; 163:743-6. [PubMed: 3554343]

3. Vogel P, Laing FC, Jeffrey RB Jr, Wing VW. Deep venous thrombosis of the lower extremity: US evaluation. Radiology. 1987; 163:747-51. [PubMed: 3554344]

4. Wells PS, Anderson DR, Rodger M, Forgie M, Kearon C, Dreyer J, et al. Evaluation of D-dimer in the diagnosis of suspected deep-vein thrombosis. N Engl J Med. 2003; 349:1227-35. [PubMed: 14507948]

5. Hou H, Ge Z, Ying P, Dai J, Shi D, Xu Z, et al. Biomarkers of deep venous thrombosis. J Thromb Thrombolysis. 2012; 34:335-46. [PubMed: 22528325]

6. Andrews EJ Jr, Fleischer AC. Sonography for deep venous thrombosis: current and future applications. Ultrasound Q. 2005; 21:213-25. [PubMed: 16344726]

7. Barnes DM, Wakefield TW, Rectenwald JE. Novel biomarkers associated with deep venous thrombosis: a comprehensive review. Biomark Insights. 2008; 3:93-100. [PubMed: 19578498]

8. Ley K. The role of selectins in inflammation and disease. Trends Mol Med. 2003; 9:263-8. [PubMed: 12829015]

9. McEver RP. Adhesive interactions of leukocytes, platelets, and the vessel wall during hemostasis and inflammation. Thromb Haemost. 2001; 86:746-56. [PubMed: 11583304] 
10. Wakefield TW, Myers DD, Henke PK. Mechanisms of venous thrombosis and resolution. Arterioscler Thromb Vasc Biol. 2008; 28:387-91. [PubMed: 18296594]

11. Furie B, Furie BC. Role of platelet P-selectin and microparticle psgl-1 in thrombus formation. Trends Mol Med. 2004; 10:171-8. [PubMed: 15059608]

12. Cambien B, Wagner DD. A new role in hemostasis for the adhesion receptor P-selectin. Trends Mol Med. 2004; 10:179-86. [PubMed: 15059609]

13. Abou-Saleh H, Theoret JF, Yacoub D, Merhi Y. Neutrophil P-selectinglycoprotein-ligand-1 binding to platelet P-selectin enhances metalloproteinase 2 secretion and platelet-neutrophil aggregation. Thromb Haemost. 2005; 94:1230-5. [PubMed: 16411399]

14. Frenette PS, Denis CV, Weiss L, Jurk K, Subbarao S, Kehrel B, et al. P-selectin glycoprotein ligand 1 (psgl-1) is expressed on platelets and can mediate platelet-endothelial interactions in vivo. J Exp Med. 2000; 191:1413-22. [PubMed: 10770806]

15. Polgar J, Matuskova J, Wagner DD. The P-selectin, tissue factor, coagulation triad. J Thromb Haemost. 2005; 3:1590-6. [PubMed: 16102023]

16. Vandendries ER, Furie BC, Furie B. Role of P-selectin and psgl-1 in coagulation and thrombosis. Thromb Haemost. 2004; 92:459-66. [PubMed: 15351841]

17. Ay C, Jungbauer LV, Sailer T, Tengler T, Koder S, Kaider A, et al. High concentrations of soluble P-selectin are associated with risk of venous thromboembolism and the P-selectin thr715 variant. Clin Chem. 2007; 53:1235-43. [PubMed: 17510305]

18. Blann AD, Noteboom WM, Rosendaal FR. Increased soluble P-selectin levels following deep venous thrombosis: cause or effect? Br J Haematol. 2000; 108:191-3. [PubMed: 10651744]

19. Rectenwald JE, Myers DD Jr, Hawley AE, Longo C, Henke PK, Guire KE, et al. D-dimer, Pselectin, and microparticles: novel markers to predict deep venous thrombosis. A pilot study. Thromb Haemost. 2005; 94:1312-7. [PubMed: 16411411]

20. Kyrle PA, Hron G, Eichinger S, Wagner O. Circulating P-selectin and the risk of recurrent venous thromboembolism. Thromb Haemost. 2007; 97:880-3. [PubMed: 17549288]

21. Ay C, Simanek R, Vormittag R, Dunkler D, Alguel G, Koder S, et al. High plasma levels of soluble P-selectin are predictive of venous thromboembolism in cancer patients: results from the Vienna Cancer and Thrombosis Study (CATS). Blood. 2008; 112:2703-8. [PubMed: 18539899]

22. Ramacciotti E, Blackburn S, Hawley AE, Vandy F, Ballard-Lipka N, Stabler C, et al. Evaluation of soluble P-selectinas a markerfor the diagnosis of deep venous thrombosis. Clin Appl Thromb Hemost. 2011; 17:425-31. [PubMed: 21593019]

23. Caprini JA. Risk assessment as a guide for the prevention of the many faces of venous thromboembolism. Am J Surg. 2010; 199(1 Suppl):S3-10. [PubMed: 20103082]

24. Kokame K, Nobe Y, Kokubo Y, Okayama A, Miyata T. Frets-vWf73, a first fluorogenic substrate for adamts13 assay. Br J Haematol. 2005; 129:93-100. [PubMed: 15801961]

25. Kremer Hovinga JA, Mottini M, Lammle B. Measurement of adamts-13 activity in plasma by the frets-vWf73 assay: comparison with other assay methods. J Thromb Haemost. 2006; 4:1146-8. [PubMed: 16689773]

26. Bombeli T, Muller M, Straub PW, Haeberli A. Cyclosporine-induced detachment of vascular endothelial cells initiates the intrinsic coagulation system in plasma and whole blood. J Lab Clin Med. 1996; 127:621-34. [PubMed: 8648267]

27. Puschel A, Lindenblatt N, Katzfuss J, Vollmar B, Klar E. Immunosuppressants accelerate microvascular thrombus formation in vivo: role of endothelial cell activation. Surgery. 2012; 151:26-36. [PubMed: 22019501]

28. Gremmel T, Ay C, Seidinger D, Pabinger I, Panzer S, Koppensteiner R. Soluble P-selectin, ddimer, and high-sensitivity c-reactive protein after acute deep vein thrombosis of the lower limb. J Vasc Surg. 2011; 54:48S-55S. [PubMed: 21890302]

29. Kvasnicka J, Horak J, Zenahlikova Z, Kvasnicka T, Simek S, Kovarnik T, et al. Reduced thrombin generation and soluble P-selectin after intravenous enoxaparin during PCI. Cardiovasc Drugs Ther. 2011; 25:243-50. [PubMed: 21584633]

30. Joffe HV, Kucher N, Tapson VF, Goldhaber SZ. Upper-extremity deep vein thrombosis: A prospective registry of 592 patients. Circulation. 2004; 110:1605-11. [PubMed: 15353493] 
31. Marshall PS, Cain H. Upper extremity deep vein thrombosis. Clin Chest Med. 2010; 31:783-97. [PubMed: 21047583]

32. Douma RA, Tan M, Schutgens R, Bates SM, Perrier A, Legnani C, et al. Using an age-dependent d-dimer cut-off value increases the number of older patients in whom deep vein thrombosis can be safely excluded. Haematologica. 2012; 97:1507-13. [PubMed: 22511491] 


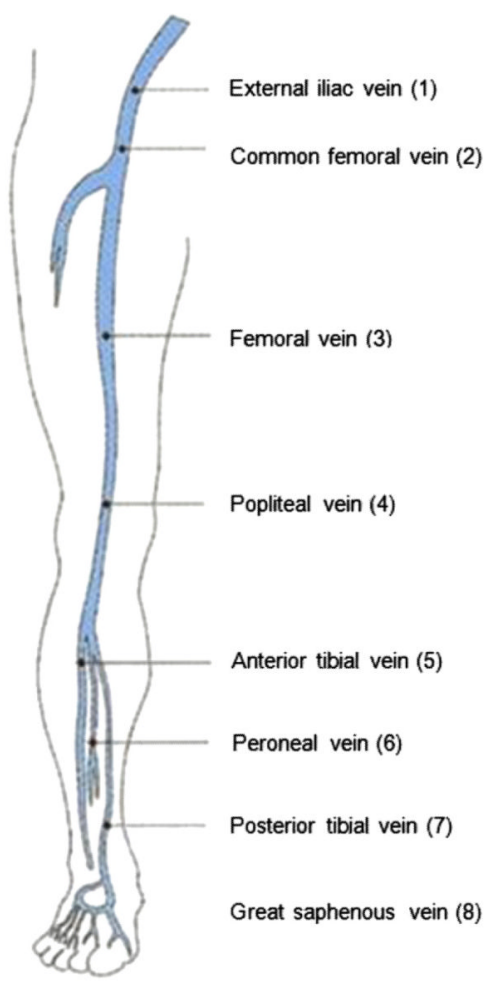

Fig 1.

Lower extremity (LE) venous anatomy divided into eight quantifiable segments. 


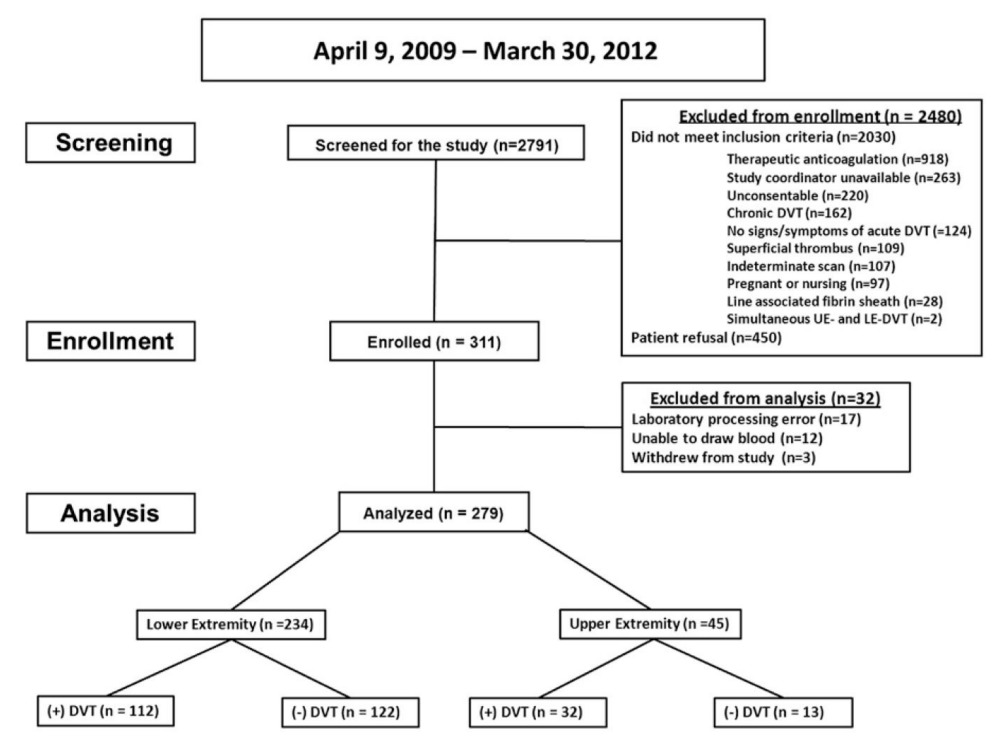

Fig 2.

Consort diagram. $D V T$, Deep venous thrombosis; $L E$, lower extremity; $U E$, upper extremity. 


\section{Table I}

Wells score for predicting the pretest probability of DVT

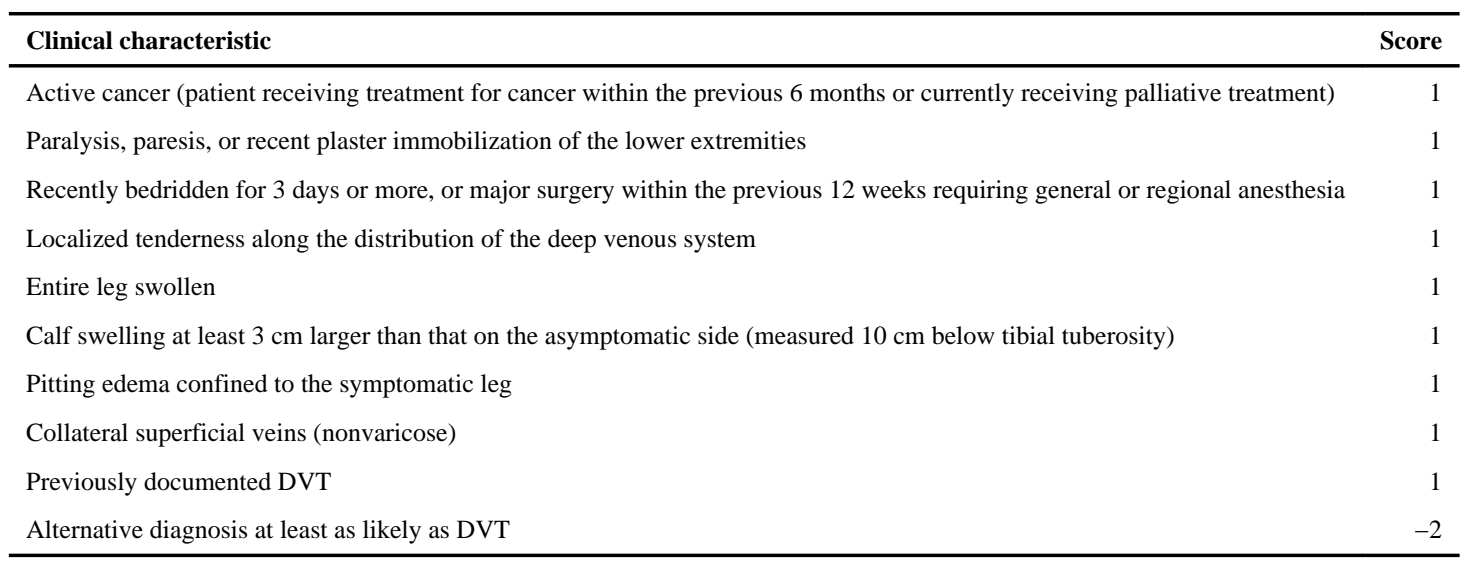

$D V T$, Deep venous thrombosis. 
Table II. A

Demographics: LE-DVT

\begin{tabular}{lccc}
\hline Variable & Negative DVT & Positive DVT & P value \\
\hline Number of patients & 122 & 112 & \\
Age, years & 53.6 & 58.3 & .016 \\
Female gender, n (\%) & $76 / 122(62)$ & $46 / 112(41)$ & .002 \\
Race (\% Caucasian) & $102 / 122(84)$ & $103 / 112(92)$ & .073 \\
Body mass index, kg/m ${ }^{2}$ (range) & $29.1(28.1-30.2)$ & $30.2(28.7-31.7)$ & .25 \\
Active smoker (\%) & $14 / 122(11)$ & $15 / 112(13)$ & .69 \\
Previous DVT (\%) & $10 / 122(8)$ & $41 / 112(37)$ & $<.001$ \\
Family history (\%) & $20 / 122(16)$ & $26 / 112(23)$ & .25 \\
Prophylactic anticoagulation (\%) & $6 / 122(5)$ & $17 / 112(15)$ & .014 \\
Active cancer (\%) & $22 / 122(18)$ & $39 / 112(35)$ & .004 \\
History of cancer (\%) & $40 / 122(33)$ & $55 / 112(49)$ & .01 \\
Immunosuppression (\%) & $15 / 122(12)$ & $21 / 112(19)$ & .21 \\
Inpatient (\%) & $7 / 122(6)$ & $26 / 112(23)$ & $<.001$ \\
Travel (\%) & $15 / 122(12)$ & $10 / 112(9)$ & .53 \\
\hline
\end{tabular}

$D V T$, Deep venous thrombosis; $L E$, lower extremity. 
Table II. B

Demographics: UE-DVT

\begin{tabular}{lccc}
\hline Variable & Negative DVT & Positive DVT & P value \\
\hline Number of patients & 13 & 32 & \\
Age, years & 49.8 & 54.9 & .39 \\
Female gender (\%) & $10 / 13(77)$ & $12 / 32(38)$ & .02 \\
Race (\% Caucasian) & $12 / 13(92)$ & $30 / 32(94)$ & .86 \\
Body mass index, $\mathrm{kg} / \mathrm{m}^{2}$ (range) & $29.8(26.0-33.7)$ & $26.5(24.2-28.5)$ & .12 \\
Active smoker (\%) & $2 / 13(15)$ & $6 / 32(19 \%)$ & .78 \\
Previous DVT (\%) & $1 / 13(8)$ & $9 / 32(28)$ & .27 \\
Family history (\%) & $2 / 13(15)$ & $3 / 32(9)$ & .95 \\
Prophylactic anticoagulation (\%) & $0 / 13(0)$ & $7 / 32(22)$ & .09 \\
Active cancer (\%) & $4 / 13(31)$ & $12 / 32(38)$ & .93 \\
History of cancer (\%) & $7 / 13(54)$ & $17 / 32(53)$ & .96 \\
Immunosuppression (\%) & $3 / 12(25)$ & $5 / 32(16)$ & .78 \\
Inpatient (\%) & $2 / 13(15)$ & $15 / 32(47)$ & .08 \\
Travel (\%) & $1 / 13(8)$ & $0 / 32(0)$ & .29 \\
\hline
\end{tabular}

$D V T$, Deep venous thrombosis; $U E$, upper extremity. 


\section{Table III. A}

Biomarkers: LE-DVT

\begin{tabular}{lccc}
\hline Biomarker & Negative DVT (95\% CI) & Positive DVT (95\% CI) & P value \\
\hline Soluble P-selectin, ng/mL & $54.5(49.9-59.2)$ & $77.2(70.7-83.8)$ & $<.001$ \\
D-dimer, mg/L & $1.04(0.76-1.33)$ & $5.67(4.89-6.44)$ & $<.001$ \\
C-reactive protein, $\mu \mathrm{g} / \mathrm{mL}$ & $1.51(0.91-2.11)$ & $5.29(4.12-6.46)$ & $<.001$ \\
von Willebrand factor $(\%$ activity) & $113.2(99.6-126.6)$ & $151.1(137.6-164.7)$ & $<.001$ \\
ADAMTS-13 (\% activity) & $102(98-107)$ & $91(86-95)$ & $<.001$ \\
Wells score & $1.72(1.54-1.92)$ & $2.88(2.62-3.13)$ & $<.001$ \\
Caprini score & $3.9(3.5-4.3)$ & $6.3(5.8-6.8)$ & $<.001$ \\
\hline
\end{tabular}

$A D A M T S$-13, A disintegrin and metalloproteinase with a thrombospondin type 1 motif, member 13 ; $C I$, confidence interval; $D V T$, deep venous thrombosis; $L E$, lower extremity. 
Table III. B

Biomarkers: UE-DVT

\begin{tabular}{lccc}
\hline Biomarker & Negative DVT (95\% CI) & Positive DVT (95\% CI) & P value \\
\hline Soluble P-selectin, $\mathrm{ng} / \mathrm{mL}$ & $50.0(34.3-65.7)$ & $59.2(49.2-69.1)$ & .3 \\
D-dimer, mg/L & $1.37(0.44-2.30)$ & $5.30(3.74-6.87)$ & $<.001$ \\
C-reactive protein, $\mu \mathrm{g} / \mathrm{mL}$ & $0.69(0.21-1.17)$ & $4.81(2.99-6.63)$ & $<.001$ \\
von Willebrand factor (\% activity) & $104.7(74.5-134.8)$ & $147.9(112.9-182.9)$ & .06 \\
ADAMTS-13 (\% activity) & $96(82-110)$ & $84(73-95)$ & .15 \\
Wells score & $1.08(0.62-1.54)$ & $2.40(1.92-2.89)$ & $<.001$ \\
\hline
\end{tabular}

ADAMTS-13, A disintegrin and metalloproteinase with a thrombospondin type 1 motif, member $13 ; C I$, confidence interval; $D V T$, deep venous thrombosis; $U E$, upper extremity. 
Table IV

Comparison of biomarkers between LE- and UE-DVTs

\begin{tabular}{lccc}
\hline Biomarker & Positive LE-DVT (95\% CI) & Positive UE-DVT (95\% CI) & P value \\
\hline Soluble P-selectin, ng/mL & $77.2(70.7-83.8)$ & $59.2(49.2-69.1)$ & .003 \\
D-dimer, mg/L & $5.67(4.89-6.44)$ & $5.30(3.74-6.87)$ & .67 \\
C-reactive protein, $\mu \mathrm{g} / \mathrm{mL}$ & $5.29(4.12-6.46)$ & $4.81(2.99-6.63)$ & .66 \\
von Willebrand factor $(\%$ activity) & $151.1(137.6-164.7)$ & $147.9(112.9-182.9)$ & .45 \\
ADAMTS-13 (\% activity) & $91(86-95)$ & $84(73-95)$ & .26 \\
Wells score & $2.88(2.62-3.13)$ & $2.40(1.92-2.89)$ & .09 \\
Caprini score & $6.3(5.8-6.8)$ & $6.1(5.1-7.8)$ & .84 \\
\hline
\end{tabular}

$A D A M T S-13$, A disintegrin and metalloproteinase with a thrombospondin type 1 motif, member $13 ; C I$, confidence interval; $D V T$, deep venous thrombosis; $L E$, lower extremity; $U E$, upper extremity. 
Table V

Comparison of LE-DVT by location: Proximal versus distal segments

\begin{tabular}{lccccc}
\hline Biomarker & Proximal segments & Distal segments & Both segments & P value & P value \\
\hline Soluble P-selectin, ng/mL & 57.5 & 75.7 & 80.6 & .10 & .47 \\
D-dimer, mg/L & 5.60 & 4.86 & 6.32 & .66 & .072 \\
C-reactive protein, $\mu \mathrm{g} / \mathrm{mL}$ & 4.49 & 3.80 & 6.51 & .41 & .025 \\
von Willebrand factor (\% activity) & 119.4 & 143.6 & 159.6 & .19 & .25 \\
ADAMTS-13 (\% activity) & 89 & 95 & 88 & .87 & .12 \\
Wells score & 1.7 & 2.7 & 3.2 & .005 & .04 \\
\hline
\end{tabular}

$A D A M T S-13$, A disintegrin and metalloproteinase with a thrombospondin type 1 motif, member $13 ; D V T$, deep venous thrombosis; $L E$, lower extremity.

${ }^{a}$ Comparison of proximal segments and all segments.

${ }^{b}$ Comparison of distal segments and all segments. 
Table VI

Effects of anticoagulation on LE-DVT biomarker levels

\begin{tabular}{|c|c|c|c|c|}
\hline Biomarker & $\begin{array}{c}\text { No prophylactic } \\
\text { anticoagulation }(95 \% \text { CI })\end{array}$ & $\begin{array}{c}\text { Prophylactic } \\
\text { anticoagulation }(95 \% \text { CI })\end{array}$ & $\begin{array}{c}\text { Therapeutic } \\
\text { anticoagulation }\end{array}$ & $\mathbf{P}$ value ${ }^{a}$ \\
\hline Number of patients & 95 & 17 & 5 & \\
\hline Soluble P-selectin, ng/mL & $77.3(70.2-84.5)$ & $76.6(58.6-94.7)$ & 44.6 & .94 \\
\hline D-dimer, ng/L & $5.50(4.65-6.35)$ & $6.62(4.63-8.62)$ & 0.99 & .29 \\
\hline C-reactive protein, $\mu \mathrm{g} / \mathrm{mL}$ & $4.98(3.78-6.17)$ & $7.03(2.85-11.22)$ & 7.13 & .33 \\
\hline von Willebrand factor ( $\%$ activity) & $158.5(133.4-162.3)$ & $183.5(129.0-211.0)$ & 179 & .31 \\
\hline ADAMTS-13 (\% activity) & $92(87-96)$ & $85(70-101)$ & & .42 \\
\hline Wells score & $2.91(2.62-3.19)$ & $2.71(2.14-3.27)$ & & .52 \\
\hline Body mass index & $29.9(28.4-31.4)$ & $32.0(26.7-37.2)$ & & .43 \\
\hline Caprini score & $6.0(5.5-6.5)$ & $8.1(6.5-9.7)$ & & .01 \\
\hline
\end{tabular}

$A D A M T S-13$, A disintegrin and metalloproteinase with a thrombospondin type 1 motif, member $13 ; C I$, confidence interval; $D V T$, deep venous thrombosis; $L E$, lower extremity.

${ }^{a}$ Comparison between patients on prophylactic anticoagulation and patients on no prophylactic or therapeutic anticoagulation. 
Table VII

Diagnosing LE-DVT

\begin{tabular}{lcccc}
\hline Biomarker & Specificity & Sensitivity & Positive predictive value & Negative predictive value \\
\hline sP-sel $\searrow 90 \mathrm{ng} / \mathrm{mL}+$ Wells $\geq 2$ & $97.5 \%$ & $27.4 \%$ & $91 \%$ & $\mathrm{NA}$ \\
D-dimer $2500 \mathrm{ng} / \mathrm{mL}+$ Wells $\geq 2$ & $65.5 \%$ & $82.3 \%$ & $69 \%$ & $\mathrm{NA}$ \\
sP-sel $\$ 60 \mathrm{ng} / \mathrm{mL}+$ Well's $<2$ & $31.1 \%$ & $91.1 \%$ & $\mathrm{NA}$ & $79 \%$ \\
D-dimer $500 \mathrm{ng} / \mathrm{mL}+$ Wells $<2$ & $29.5 \%$ & $98.2 \%$ & $\mathrm{NA}$ & $95 \%$ \\
\hline
\end{tabular}

$D V T$, Deep venous thrombosis; $L E$, lower extremity; $s P$-sel, soluble P-selectin. 